\title{
Proceedings
}

Juntendo Medical Journal

2016. 62 (Suppl 1), 166

\section{The Effect of Cooling on Muscle Strength and Muscle Cross-Sectional Area During Detraining}

\author{
SHINYA ENDO*1), KEISHOKU SAKURABA*1) 2), ATSUSHI KUBOTA*2), JiRO SHIKAKURA*2) \\ *1) Graduate School of Health and Sports Science, Juntendo University, Chiba, Japan, *2) Faculty of Health and Sports \\ Science, Juntendo University, Chiba, Japan
}

Two weeks of detraining produces $6.4 \%$ of muscle atrophy even after long-term strength training. Thus, to maintain the training effect, preventing the muscle weakness and muscle atrophy caused by detraining would be valuable. Exposure to cold stimulus by cold water inhibits muscular atrophy.

The purpose of this study was to investigate the effect of cooling by ice bag on muscle weakness and muscle atrophy caused by detraining.

Six healthy males $(23.0 \pm 2.5 \mathrm{y} / \mathrm{o})$ performed elbow flexion resistance training three times per week for 6 weeks. After the 6 weeks training, the subjects were instructed to limit upper arm activity within their activity of daily living level during three weeks of detraining period. During the de-training period, one of the arms was cooled at inside of upper arm by an ice bag for $30 \mathrm{~min}$ a day (ICE), and the other arms was control condition (CON). The measurements were elbow flexion torques at angular speeds of 60 and $120 \mathrm{deg} / \mathrm{s}$ under concentric contraction (CC60, CC120) and isometric contraction (IM), cross-sectional area (CSA) and circumference of the upper arm. The measurements were done, at pre-training (PRE), post-training (POST), after the first week (D1), second week (D2) and third week (D3) of detraining.

IM significantly increased after training in both conditions $(\mathrm{p}<0.05)$. Significant decreases of IM in CON was observed at D2 $(66.6 \pm 17.4 \mathrm{~N} \cdot \mathrm{m})$ compared to POST $(78.8 \pm 24.8 \mathrm{~N} \cdot \mathrm{m})(\mathrm{p}<0.05)$. Circumference significantly increased after training in both conditions (CON: $102.1 \pm 1.0 \%$, ICE: $101.4 \pm 0.7 \%)(\mathrm{p}<0.05)$. Significant decreases of circumference in CON was observed at D3 (29.6 $\pm 3.4 \mathrm{~cm})$ compared to $\mathrm{D} 2(29.8 \pm 3.5 \mathrm{~cm})(\mathrm{p}<0.05)$. CSA significantly increased after training in both conditions $(\mathrm{CON}$ : $108.5 \pm 3.3 \%$, ICE: $107.9 \pm 2.2 \%) \quad(\mathrm{p}<0.05)$. CSA significantly decreased after the detraining in both conditions. However, there was no significant difference in all of measurements between $\mathrm{CON}$ and ICE.

Only IM increased after the training. It may be because of the fact that the training was composed of isometric contraction. Even though increased the muscle force, circumference and CSA by the training decreased by the detraining. On the other hand, there was no decrease in IM and circumference in ICE during detraining period. This result suggests the possibility that the cooling prevents muscle weakness and muscle atrophy; however, there was no significant difference between both conditions, therefore we consider that the effect of cooling was weak. In conclusion, the cooling for $30 \mathrm{~min}$ a day shows possibility that the cooling prevents muscle weakness and muscle atrophy caused by detraining.
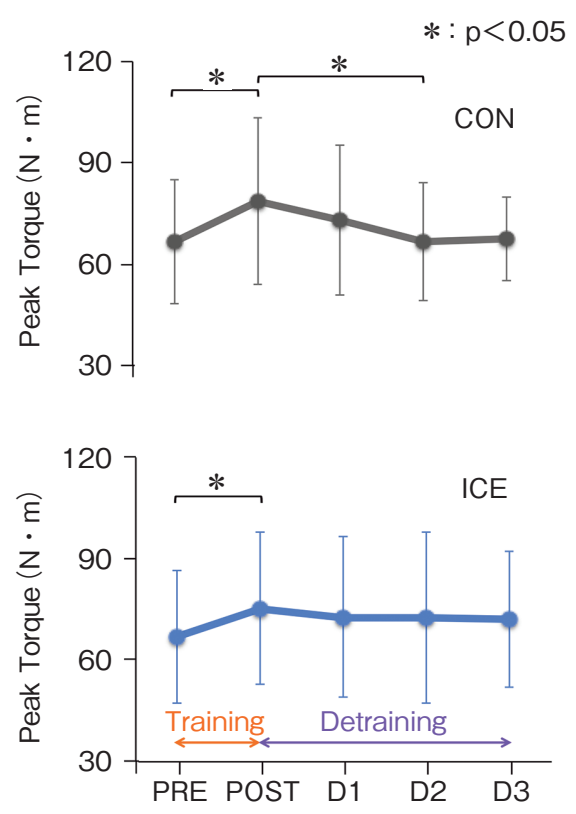

Corresponding author: Keishoku Sakuraba

Graduate School of Health and Sports Science, Juntendo University

1-1 Hirakagakuendai, Inzai-shi, Chiba 270-1695, Japan

TEL: +81-476-98-1001 E-mail: sakuraba@juntendo.ac.jp

The $2^{\text {nd }}$ Congress, International Academy of Sportology 〔Held on Sep. 12, 2015〕

〔Received Dec. 18, 2015〕 